\title{
LT@Helsinki at SemEval-2020 Task 12: Multilingual or language-specific BERT?
}

Marc Pàmies

\author{
Emily Öhman Kaisla Kajava \\ University of Helsinki \\ firstname.lastname@helsinki.fi
}

Jörg Tiedemann

\begin{abstract}
This paper presents the different models submitted by the LT@Helsinki team for the SemEval 2020 Shared Task 12. Our team participated in sub-tasks A and C; titled offensive language identification and offense target identification, respectively. In both cases we used the so-called Bidirectional Encoder Representation from Transformer (BERT), a model pre-trained by Google and fine-tuned by us on the OLID and SOLID datasets. The results show that offensive tweet classification is one of several language-based tasks where BERT can achieve state-of-the-art results.
\end{abstract}

\section{Introduction}

The number of social media users has reached 3.5 billion, and an average of 6,000 tweets are generated every second (Mathew et al., 2019) . With such a large volume of tweets, it seems inevitable that some use offensive language. A study from 2014 found that $67 \%$ of social media users had been exposed to online hate, and $21 \%$ had been the target of online hate (Oksanen et al., 2014).

The usual approach on social media sites is to forbid hate speech in the terms of service and to censor any inappropriate content detected by their algorithms, but still, companies like Facebook or Twitter have been harshly criticized for not doing enough (Del Vigna et al., 2017). This criticism has forced companies to try and find accurate and scalable solutions that solve the problem of offensive language detection using automated methods. Workshops dealing with offensive language, such as TRAC (Kumar et al., 2018b), TA-COS (Lefever et al., 2018), or ALW1 (Waseem et al., 2017), as well as shared tasks like GermEval (Wiegand et al., 2018), TRAC-1 (Kumar et al., 2018a), and OffensEval 2019 (Zampieri et al., 2019b), are becoming more and more prevalent.

The task we address here, SemEval 2020 task 12 (Zampieri et al., 2020), is titled Multilingual Offensive Language Identification in Social Media and is divided into the following sub-tasks:

A. Offensive Language Identification in several languages (Arabic, Danish, English, Greek, Turkish): whether a tweet is offensive or not.

B. Categorization of Offense Types: whether an offensive tweet is targeted or untargeted.

C. Offense Target Identification: whether a targeted offensive tweet is directed towards an individual, a group or otherwise.

In this paper the system created by the LT@Helsinki team for sub-tasks A and C will be described. In sub-task $\mathrm{A}$ we participated in all the language tracks. For sub-task $\mathrm{C}$ the only language available was English. We qualified as second in sub-task $\mathrm{C}$ and our submission for sub-task A ranked first for Danish, seventh for Greek, eighteenth for Turkish, and forty-sixth for Arabic. In all submissions we used BERT-Base models (Devlin et al., 2019) fine-tuned on each dataset provided by the task organizers. We also experimented with random forest with TF-IDF and other kinds of features, but the results on the development set were not as good as with transfer learning techniques based on pre-trained language models. We discovered that, at least for this data, the language-specific model worked better than the multilingual.

This work is licensed under a Creative Commons Attribution 4.0 International License. License details: http:// creativecommons.org/licenses/by/4.0/. 


\section{Background}

All the data used to train our classifiers was provided by the OffensEval organizers (Zampieri et al., 2019a). The tweets were retrieved from the Twitter Search API and manually labeled by at least two human annotators. As a pre-processing step, they desensitized all tweets replacing usernames and website URLs by general tokens. The following datasets were used for the offensive language identification problem:

\begin{tabular}{l|ccc} 
Language & Training & Test & Total \\
\hline Arabic & 8,000 & 2,000 & 10,000 \\
Danish & 2,960 & 329 & 3,289 \\
Greek & 8,743 & 1,544 & 10,287 \\
Turkish & 31,756 & 3,528 & 35,284
\end{tabular}

Table 1: Sub-task A datasets

Some of the algorithms that can be found in the literature are random forest (Burnap and Williams, 2015), logistic regression (Davidson et al., 2017), and Support Vector Machine (Malmasi and Zampieri, 2018), as well as deep learning approaches like Convolutional Neural Networks (Gambäck and Sikdar, 2017) or Convolutional-GRU (Zhang et al., 2018). However, in 2018 deep pre-trained language models obtained state-of-the-art results in several NLP downstream tasks, text classification being one of them. In particular, Google's Bidirectional Encoder Representations from Transformers (BERT) (Devlin et al., 2019) stood above the rest for being deeply bidirectional and using the novel self-attention layers from the transformer model (Vaswani et al., 2017), which allows it to better understand a word's context by looking at both its left and right neighbours. BERT provides out-of-the-box pre-trained monolingual and multilingual models that, after massive training on general corpora, can be fine-tuned with a small amount of task-specific data and still offer excellent performance. The results published in OffensEval's previous edition (Zampieri et al., 2019b) proved that BERT is well suited for the offensive language detection task, since it was the chosen method of most of the top teams, including the winners of subtasks A (Liu et al., 2019) and C (Nikolov and Radivchev, 2019).

For the Danish dataset (Sigurbergsson and Derczynski, 2020) we used Nordic BERT ${ }^{1}$, which is pretrained on Danish Wikipedia texts, Danish text from Common Crawl, Danish OpenSubtitles, and text from popular Danish online forums. All in all, the training corpus consists of over 90M sentences and almost 20M unique tokens. For the other languages we used the standard BERT-Base models (Devlin et al., 2019) with no further pre-training and only the provided datasets for each language, i.e. Arabic (Mubarak et al., 2020), Greek (Pitenis et al., 2020), and Turkish (Çöltekin, 2020).

In sub-task C, since the language was English it was possible to use the Offensive Language Identification Dataset (OLID), provided by the organizers of OffensEval 2019. Despite consisting of different sub-tasks, all of them shared the same dataset that was annotated according to a three-level hierarchical model, so that each sub-task could use as dataset a subset of the previous sub-task's dataset. First, all tweets were labeled as either offensive (OFF) or not offensive (NOT). Then, for sub-task B, all the offensive tweets were labeled as targeted (TIN) or untargeted insults (UNT). And finally, for the last sub-task, the third level of the hierarchy labeled targeted insults based on who was the recipient of the offense: an individual (IND), a group (GRP) or a different kind of entity (OTH). To illustrate this, Figure 2 displays OLID's label distribution.

The corpus from sub-task C in OffensEval 2019 contains 4,089 English tweets, of which 3,876 originally belonged to the training set and the remaining 213 to the test set (Figure 2). We also had at our disposal over 9M English tweets provided by the OffensEval 2020 organizers (Rosenthal et al., 2020). However, these were processed by unsupervised learning methods instead of human annotators, which is why on this occasion each tweet was not associated with a label but given two values: (1) the confidence that it belongs to a specific class and (2) its standard deviation.

\footnotetext{
${ }^{1}$ https://github.com/botxo/nordic_bert
} 


\begin{tabular}{cccrr|r}
\hline A & B & C & Training & Test & Total \\
\hline OFF & TIN & IND & 2,407 & 100 & 2,507 \\
OFF & TIN & GRP & 1,074 & 78 & 1,152 \\
OFF & TIN & OTH & 395 & 35 & 430 \\
OFF & UNT & - & 524 & 27 & 551 \\
NOT & - & - & 8,840 & 620 & 9,460 \\
\hline All & & & 13,240 & 860 & 14,100 \\
\hline
\end{tabular}

Table 2: Distribution of label combinations in OLID.

\section{System Overview and Experimental Setup}

In this section we discuss the system we created and setup we used for subtask A and subtask C.

\subsection{Sub-task A}

For the binary classification problem (sub-task A) two baseline methods were implemented and evaluated on the training Danish dataset: random forest and BERT.

For the random forest implementation, the pre-processing steps were lower-casing all characters, removing irrelevant punctuation marks, reducing the length of characters that appear more than two consecutive times, and converting hashtags to sentences by adding white spaces before every capital letter. Tokenization was done with the TweetTokenizer tool from NLTK. The same library was also used to perform stopword removal and word stemming. Emojis were removed after storing their "sentiment score" as a feature employing the emosent Python utility package (Novak et al., 2015). We also used surface-level features such as the number of URL tokens and @USER mentions, the total number of characters, punctuation marks and words in each post, average word length, percentage of capital letters, and the number of abusive terms. A ratio of 10:1 was applied when splitting the dataset into training and validation sets. Since the Danish dataset was relatively small, 10-fold cross-validation was done to obtain reliable results. Otherwise the F1 scores relied too much on which samples would fall in the validation set. The random forest implementation from scikit-learn (Pedregosa et al., 2011) was used. The optimal parameters were found with grid search.

\begin{tabular}{lr} 
System & macro-F1 \\
\hline All NOT & 0.465 \\
Random Forest with TFIDF & 0.773 \\
Multilingual BERT-Base & 0.768 \\
Nordic BERT & 0.804
\end{tabular}

Table 3: Development results sub-task A, Danish dataset

The other approach was to apply pre-trained BERT models. After experimenting with both the original Base version (12-layer, 768-hidden, 12-heads, $110 \mathrm{M}$ parameters) and the publicly available Nordic BERT $^{2}$ it was clear that the second one was better suited for the task. This shows that further pre-training of BERT can significantly boost performance, especially in cases like this where there is very little data (the Danish dataset was by far the smallest of the shared task). Our final submission was generated by the Danish version of Nordic BERT fine-tuned on the OffensEval 2020 data, using a batch size of 32 for training and 16 for both validation and testing. The learning rate of Adam optimizer was set to 2e-5 and the model was trained for 4 epochs. The sequence length was set to 128 because, even though some instances are very long (some of them are not tweets but Reddit comments), an analysis of the length distribution showed that only $3.4 \%$ of the training examples reached the limit after being tokenized by BertTokenizer.

\footnotetext{
${ }^{2}$ https://github.com/botxo/nordic_bert
} 
Due to time constraints we were not able to experiment in depth with all languages available for subtask A, which is why Danish was our main focus. However, seeing that BERT could perform so well with almost no pre-processing, we decided to generate results for the other three languages as well. For Turkish and Arabic, we used the BERT-base-multilingual-cased model with a maximum sequence length of 128, a batch size of 32, and a learning rate of 2e-5. On the other hand, for Greek we used the BERTbase-uncased model after lowercasing and translating the entire dataset into English. In all cases we trained the model for 4 epochs, used BertTokenizer to tokenize the tweets, and padded and truncated the sequences to make sure each data instance had the same length.

\subsection{Sub-task C}

The target identification problem (sub-task C, English) had an additional level of difficulty with respect to the first task because the dataset was highly imbalanced and composed of three classes instead of two. Thus, in this case we focused our efforts on balancing the given dataset to prevent having a high number of misclassifications from instances of the minority class (which would notably affect the resulting macro-F1 score). In order to overcome the class imbalance problem, we trained our model on all the data from 2019 and some additional instances from the non-majority classes (GRP and OTH) of the 2020 dataset. Only the $300 \mathrm{OTH}$ instances and 237 GRP instances of highest confidence were added in order to slightly increase the balance of the dataset. We experimented with different thresholds to select more samples but at the end we decided to keep the value low to ensure that all tweets used for training are tagged correctly. Finally, to overcome the class imbalance problem, an over-sampling technique with replacing was applied. We simply produced copies of instances from the minority classes to end up with a totally balanced dataset of 11,628 tweets (3,876 for each class). Under-sampling was not an option because then we would be facing a data scarcity problem, and we experimented with ratios other than 1:1:1 obtaining promising results but not significantly better than the proposed approach. Another interesting approach, which was chosen by the winners of last year's edition (Nikolov and Radivchev, 2019), is to modify the classification thresholds (i.e. lower the thresholds for classes OTH and GRP) to get less new examples classified as the majority class.

Then, the now balanced dataset was used as input for a BERT-base-uncased model with a maximum sequence length of 128 and batch sizes of 32,16 and 8 for training, validation and prediction respectively. The learning rate was set to $2 \mathrm{e}-5$ and the training lasted 4 epochs. In this case we did not perform any preprocessing step other than lowercasing since none of the attempts (i.e. translating emojis to sentences, splitting hashtags into separate words etc.) significantly boosted performance. Moreover, we believe that for this specific task it might not be a good idea to remove @ USER mentions or certain stop words since they can carry valuable information for target identification.

\subsection{Unfruitful Attempts at Performance Improvement}

\begin{tabular}{|l|l|l|l|l|l|l|l|l|l|l|}
\hline & pos & neg & ang & ant & dis & fea & joy & sad & sur & tru \\
\hline NOT & 51.476 & 79.136 & 30.028 & 17.623 & 29.685 & 47.055 & 16.588 & 25.843 & 10.753 & 26.785 \\
\hline OFF & 68.640 & 110.350 & 41.481 & 23.474 & 37.543 & 58.420 & 22.374 & 46.046 & 9.190 & 26.838 \\
\hline
\end{tabular}

Table 4: Emotion word distribution in offensive and non-offensive messages.

Offensive tweets and posts had statistically significant amounts of emotion-laden words, including positive ones. We arrived at these results by augmenting the data (see Ohman (2016)) using the NRC Emotion Lexicon (Mohammad and Turney, 2013) for the languages in question and then comparing the normalized word counts per offensive post. We did not take context or negation into account. Unfortunately, we were unable to meaningfully utilize this information to improve the performance of our system. Even though offensive tweets were more likely to include emotion words and more of them, a significant number of offensive tweets contained no emotion words and many non-offensive tweets did contain them. Nonetheless, the results were quite interesting so we wanted to share them here in the hopes that they might be useful to someone else, perhaps for the OffensEval 2021 tasks. The numbers 
represent normalized word counts for words classified as containing a specific sentiment or emotion for data from the Danish dataset.

\section{Results}

For Danish, using Nordic BERT for the final submission, we obtained an accuracy of $92.38 \%$ and F1score of $81.18 \%$ (Figure 1 a). The confusion matrix shows that only 9 out of 34 offensive tweets (OFF) were misclassified, and 16 out of 294 not offensive tweets (NOT) were wrongly classified as offensive. Regarding the other languages, for Greek we obtained an F1-score of 82.6\%, 77.2\% for Turkish, and $73.1 \%$ for Arabic.

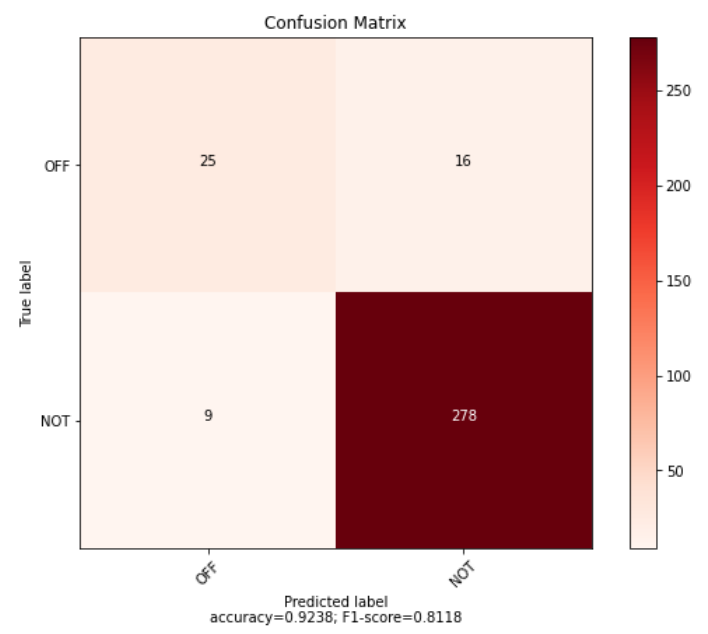

(a) Danish dataset Sub-Task A

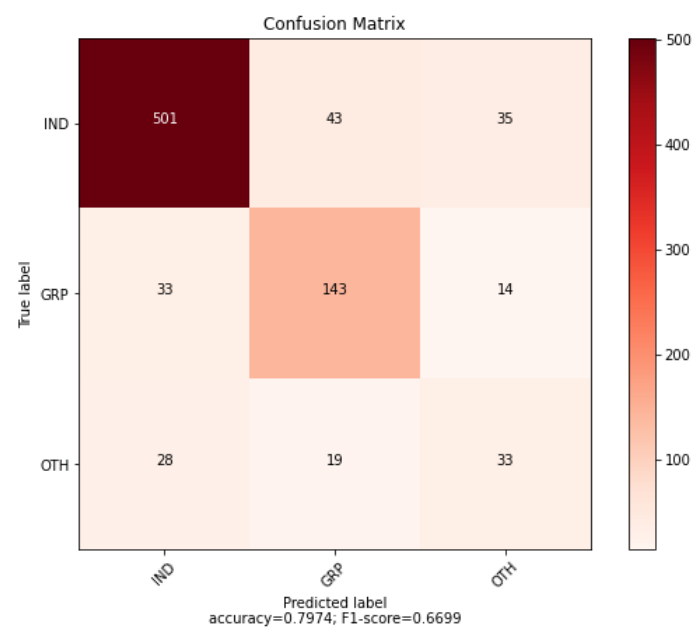

(b) Sub-Task C

Figure 1: Official results of the LT@Helsinki Team

In sub-task C our submission ranked second with an accuracy of $79.74 \%$ and F1-score of $66.99 \%$. The relatively low F1-score is due to the high numbers of misclassifications of the minority class (only 33 OTH instances were correctly classified out of 82) (see Figure $1 \mathrm{~b}$ ). Despite our efforts in balancing the dataset, it seems like the skewed class distribution inevitably added bias to the model.

It is impressive how good BERT performed overall even though we applied very few pre-processing steps to the data. Moreover, the results obtained on previously unseen data indicate that none of our models were heavily overfitted.

\section{Conclusions}

Our scores on evaluation data show that models pre-trained on general corpora can obtain competitive results when there is very little data available, as was the case of the Danish sub-task where we obtained the best results. BERT performed well on both tasks, but there is still room for improvement. In the future, we intend to experiment with those languages that we could not focus on this time. It should be noted that multilingual BERT works best with languages similar to English (Pires et al., 2019) so it is very likely that the other languages would have benefited from the use of language-specific models even more than Danish as Danish is the language most similar to English in comparison to Greek, Turkish, and Arabic.

A more thorough comparison between multilingual and language-specific BERT would yield more definitive answers as to whether language-specific is always best or whether that is language-dependent. Further examination and use of emotion-laden words in offensive texts could also help with the detection of offensive texts. Examining other baseline methods and combining them into an ensemble model with majority voting is another approach to consider for future work. With regards to the class imbalance problem, other techniques such as adjusting the classification thresholds or enriching the dataset with back and forth machine translation of the minority class could prove to be useful. 


\section{References}

Pete Burnap and Matthew L Williams. 2015. Cyber hate speech on twitter: An application of machine classification and statistical modeling for policy and decision making. Policy \& Internet, 7(2):223-242.

Çağnı Çöltekin. 2020. A Corpus of Turkish Offensive Language on Social Media. In Proceedings of the 12th International Conference on Language Resources and Evaluation. ELRA.

Thomas Davidson, Dana Warmsley, Michael W. Macy, and Ingmar Weber. 2017. Automated hate speech detection and the problem of offensive language. In ICWSM.

Fabio Del Vigna, Andrea Cimino, Felice Dell'Orletta, Marinella Petrocchi, and Maurizio Tesconi. 2017. Hate me, hate me not: Hate speech detection on facebook. In Proceedings of the First Italian Conference on Cybersecurity (ITASEC17), pages 86-95.

Jacob Devlin, Ming-Wei Chang, Kenton Lee, and Kristina Toutanova. 2019. Bert: Pre-training of deep bidirectional transformers for language understanding. In NAACL-HLT.

Björn Gambäck and Utpal Kumar Sikdar. 2017. Using convolutional neural networks to classify hate-speech. In Proceedings of the first workshop on abusive language online, pages 85-90.

Ritesh Kumar, Atul Kr. Ojha, Shervin Malmasi, and Marcos Zampieri. 2018a. Benchmarking aggression identification in social media. In TRAC@COLING 2018.

Ritesh Kumar, Atul Kr Ojha, Marcos Zampieri, and Shervin Malmasi. 2018b. Proceedings of the first workshop on trolling, aggression and cyberbullying (trac-2018). In Proceedings of the First Workshop on Trolling, Aggression and Cyberbullying (TRAC-2018).

Els Lefever, Bart Desmet, and Guy De Pauw. 2018. Ta-cos 2018: 2nd workshop on text analytics for cybersecurity and online safety: Proceedings. In TA-COS 2018-2nd Workshop on Text Analytics for Cybersecurity and Online Safety, collocated with LREC 2018, 11th edition of the Language Resources and Evaluation Conference. European Language Resources Association (ELRA).

Ping Liu, Wen Li, and Liang Zou. 2019. Nuli at semeval-2019 task 6: transfer learning for offensive language detection using bidirectional transformers. In Proceedings of the 13th International Workshop on Semantic Evaluation, pages 87-91.

Shervin Malmasi and Marcos Zampieri. 2018. Challenges in discriminating profanity from hate speech. Journal of Experimental \& Theoretical Artificial Intelligence, 30(2):187-202.

Binny Mathew, Ritam Dutt, Pawan Goyal, and Animesh Mukherjee. 2019. Spread of hate speech in online social media. In Proceedings of the 10th ACM Conference on Web Science, WebSci '19, page 173-182, New York, NY, USA. Association for Computing Machinery.

Saif M. Mohammad and Peter D. Turney. 2013. Crowdsourcing a word-emotion association lexicon. 29(3):436465 .

Hamdy Mubarak, Ammar Rashed, Kareem Darwish, Younes Samih, and Ahmed Abdelali. 2020. Arabic offensive language on twitter: Analysis and experiments. arXiv preprint arXiv:2004.02192.

Alex Nikolov and Victor Radivchev. 2019. Nikolov-radivchev at semeval-2019 task 6: Offensive tweet classification with bert and ensembles. In Proceedings of the 13th International Workshop on Semantic Evaluation, pages 691-695.

Petra Kralj Novak, Jasmina Smailović, Borut Sluban, and Igor Mozetič. 2015. Sentiment of emojis. PloS one, 10(12):e0144296.

Atte Oksanen, James Hawdon, Emma Holkeri, Matti Näsi, and Pekka Räsänen. 2014. Exposure to online hate among young social media users. Sociological studies of children \& youth, 18(1):253-273.

Fabian Pedregosa, Gaël Varoquaux, Alexandre Gramfort, Vincent Michel, Bertrand Thirion, Olivier Grisel, Mathieu Blondel, Peter Prettenhofer, Ron Weiss, Vincent Dubourg, et al. 2011. Scikit-learn: Machine learning in python. Journal of machine learning research, 12(Oct):2825-2830.

Telmo Pires, Eva Schlinger, and Dan Garrette. 2019. How multilingual is multilingual bert? arXiv preprint arXiv:1906.01502. 
Zeses Pitenis, Marcos Zampieri, and Tharindu Ranasinghe. 2020. Offensive Language Identification in Greek. In Proceedings of the 12th Language Resources and Evaluation Conference. ELRA.

Sara Rosenthal, Pepa Atanasova, Georgi Karadzhov, Marcos Zampieri, and Preslav Nakov. 2020. A Large-Scale Semi-Supervised Dataset for Offensive Language Identification. In arxiv.

Gudbjartur Ingi Sigurbergsson and Leon Derczynski. 2020. Offensive Language and Hate Speech Detection for Danish. In Proceedings of the 12th Language Resources and Evaluation Conference. ELRA.

Ashish Vaswani, Noam Shazeer, Niki Parmar, Jakob Uszkoreit, Llion Jones, Aidan N Gomez, Łukasz Kaiser, and Illia Polosukhin. 2017. Attention is all you need. In Advances in neural information processing systems, pages 5998-6008.

Zeerak Waseem, Wendy Hui Kyong Chung, Dirk Hovy, and Joel Tetreault. 2017. Proceedings of the first workshop on abusive language online. In Proceedings of the First Workshop on Abusive Language Online.

Michael Wiegand, Melanie Siegel, and Josef Ruppenhofer. 2018. Overview of the GermEval 2018 Shared Task on the Identification of Offensive Language. In Proceedings of GermEval.

Marcos Zampieri, Shervin Malmasi, Preslav Nakov, Sara Rosenthal, Noura Farra, and Ritesh Kumar. 2019 a. Predicting the Type and Target of Offensive Posts in Social Media. In Proceedings of the 2019 Conference of the North American Chapter of the Association for Computational Linguistics (NAACL), pages 1415-1420.

Marcos Zampieri, Shervin Malmasi, Preslav Nakov, Sara Rosenthal, Noura Farra, and Ritesh Kumar. 2019 b. SemEval-2019 Task 6: Identifying and Categorizing Offensive Language in Social Media (OffensEval). In Proceedings of The 13th International Workshop on Semantic Evaluation (SemEval).

Marcos Zampieri, Preslav Nakov, Sara Rosenthal, Pepa Atanasova, Georgi Karadzhov, Hamdy Mubarak, Leon Derczynski, Zeses Pitenis, and Çağrı Çöltekin. 2020. SemEval-2020 Task 12: Multilingual Offensive Language Identification in Social Media (OffensEval 2020). In Proceedings of SemEval.

Ziqi Zhang, David Robinson, and Jonathan Tepper. 2018. Detecting hate speech on twitter using a convolution-gru based deep neural network. In European semantic web conference, pages 745-760. Springer.

Emily Öhman, Timo Honkela, and Jörg Tiedemann. 2016. The challenges of multi-dimensional sentiment analysis across languages. In Proceedings of the Workshop on Computational Modeling of People's Opinions, Personality, and Emotions in Social Media (PEOPLES), pages 138-142. 